\title{
A Tecnologia Assistiva e a Inclusão Educacional de Pessoas com Deficiência: um mapeamento sistemático da literatura
}

\author{
Ana Carolina C. de Melo ${ }^{1}$, Ellen Polliana R. Souza ${ }^{2}$, José Vinícius V. Lima ${ }^{1}$ \\ ${ }^{1}$ Universidade de Pernambuco - UPE \\ ${ }^{2}$ Universidade Federal Rural de Pernambuco - UFRPE \\ $\{$ accm,jvvl\}@ecomp.poli.br, ellen.polliana@gmail.com
}

\begin{abstract}
Assistive Technology (AT) becomes an important instrument of inclusion, promoting the autonomy of Person with Disability $(P w D)$ in different contexts. In this sense, this paper presents a systematic mapping of the literature, whose objective is to investigate the advances in the use of AT resources and services for the inclusion of the PwD in the educational scope, as well as to make available a catalogue with the identified digital resources. A total of 12,279 primary studies were analyzed between the years 2010 and 2019, and at the end, 104 were included in the mapping. The results identified a visual and hearing impairment with a greater amount of AT resources developed, in addition to the difficulties and benefits reported during its use.
\end{abstract}

Resumo. A Tecnologia Assistiva (TA) torna-se um importante instrumento de inclusão, promovendo a autonomia da Pessoa com Deficiência (PcD) em diferentes contextos. Neste sentido, este artigo apresenta um mapeamento sistemático da literatura, cujo objetivo é investigar os avanços no uso dos recursos e serviços de TA para a inclusão da PcD no âmbito educacional, bem como disponibilizar um catálogo com os recursos digitais identificados. Um total de 12.279 estudos primários foram analisados entre os anos de 2010 e 2019, e ao final, 104 foram incluídos no mapeamento. Os resultados identificaram um destaque as deficiências visual e auditiva com maior quantidade de recursos de TA desenvolvidos, além das dificuldades e benefícios relatados durante o seu uso.

\section{Introdução}

Os debates acerca da inclusão da Pessoa com Deficiência (PcD) enfatizam o acesso desta comunidade a serviços essenciais, como a educação, por exemplo. Frente a essa situação, mais do que conhecer as necessidades da $\mathrm{PcD}$, pesquisadores de diversas áreas, principalmente educação e informática, têm buscado meios pelos quais possam oferecer recursos de apoio ao seu processo de ensino e aprendizagem. Neste cenário, o uso da informática aliada à constante renovação de recursos desenvolvidos, torna-se um instrumento importante para o atendimento de pessoas com necessidades específicas e a sua inserção em ambientes de aprendizagem. Isso contribui para a existência de uma sociedade de participação plena e garante os princípios básicos da inclusão [Alves and Aguiar 2014].

A Tecnologia Assistiva (TA) surge como um importante recurso, uma vez que propicia a inclusão da $\mathrm{PcD}$, conferindo-a autonomia no desenvolvimento de suas atividades através de auxílios técnicos personalizados para atender suas necessidades. Bersch (2017) define TA como um termo utilizado para identificar todo o arsenal de recursos e 
serviços que contribuem para ampliar as habilidades funcionais da $\mathrm{PcD}$ e, consequentemente, promover vida independente aliada a inclusão. Os recursos são definidos como itens, produtos ou sistemas fabricados e utilizados com o objetivo de melhorar as capacidades funcionais da PcD. Já os serviços são caracterizados como àqueles que auxiliam diretamente a PcD a selecionar, adquirir ou utilizar os recursos de tecnologia assistiva.

Em consequência disso, no universo da TA, a identificação e classificação dos estudos primários com foco no desenvolvimento de recursos e serviços, mostra-se crescente na academia, tendo em vista a quantidade de trabalhos disponíveis, tanto em plataformas de artigos nacionais, quanto internacionais [Wohlin et al. 2013]. Contudo, para auxiliar o acesso dessas informações, existem estudos secundários que visam analisar estudos primários relativos a certas questões de pesquisa, promovendo aos pesquisadores uma visão geral de uma área de interesse [Kitchenham et al. 2010].

Diante da importância da inclusão de pessoas com deficiência no âmbito educacional, o presente trabalho tem como objetivo realizar um Mapeamento Sistemático da Literatura (MSL), a fim de identificar os avanços no uso de recursos e serviços de tecnologia assistiva que auxiliam na inclusão educacional da $\mathrm{PcD}$, bem como disponibilizar um catálogo online com os recursos digitais identificados na literatura. Dentre os benefícios para a realização do MSL, estão: (i) resumir e disseminar os resultados da pesquisa, promovendo uma visão abrangente da área estudada; (ii) identificar as lacunas existentes na literatura, fornecendo a oportunidade de trabalhos futuros.

Além desta seção introdutória, o trabalho está estruturado da seguinte forma: a Seção 2 exibe os trabalhos relacionados; a Seção 3 detalha o método utilizado; os resultados são discutidos na Seção 4, por fim, a Seção 5 traz as conclusões e trabalhos futuros.

\section{Trabalhos Relacionados}

Gonçalves et al. (2019) publicaram um MSL cujo objetivo era apresentar as ferramentas assistivas que fornecem apoio ao ensino de crianças com Transtorno do Espectro Autista (TEA). Um total de 17 estudos foram selecionados e, como resultados, foram identificadas 17 ferramentas, sendo a maioria para o auxílio da alfabetização. Ademais, constatou-se que, apesar do MSL não ter sido limitado por um período de tempo, o primeiro estudo publicado foi no ano de 2011, indicando que estudos na área são relativamente novos.

Freitas e Souza (2018) apresentaram uma revisão sistemática que investigou as TAs que apoiam o ensino da matemática para alunos com deficiência visual. Ao final, um total de 40 estudos foram considerados e, dentre os resultados, foram identificadas 59 tecnologias que possibilitam explorar os diversos sentidos (tátil, auditivo, háptico e multimodal) dos alunos para auxiliar no ensino dos diferentes conteúdos da matemática. Além do mais, tecnologias para leitura da matemática têm predominado nos últimos anos.

No trabalho de Neto (2015), foi realizada uma pesquisa bibliográfica manual nos anais do SBIE e WIE no período de 2001 a 2014, com o objetivo em identificar as soluções de TA que auxiliam no processo de inclusão da $\mathrm{PcD}$ na universidade e no mercado de trabalho. Ao final, 40 estudos foram considerados na pesquisa e, os achados identificaram soluções em ferramentas e softwares educativos com foco na deficiência auditiva, assim como recursos tecnológicos voltados a auxiliar na comunicação alternativa.

De forma a facilitar o entendimento dos trabalhos relacionados em relação ao tra- 
balho proposto, a Tabela 1 apresenta um resumo comparativo. Em contraste com os trabalhos supracitados, este estudo realizou dois tipos de busca: manual e automática. Como consequência, foram consideradas mais fontes de pesquisa e mais estudos primários foram analisados. Posteriormente, realizou-se a catalogação dos recursos digitais de TA identificados na literatura, os quais são voltados aos diferentes tipos de deficiência.

Tabela 1. Visão geral entre os trabalhos relacionados.

\begin{tabular}{c|c|c|c|c|c}
\hline Autor(es) & Busca & $\begin{array}{c}\text { Quant. de } \\
\text { Fontes }\end{array}$ & Deficiência & $\begin{array}{c}\text { Recursos } \\
\text { Disponíveis }\end{array}$ & $\begin{array}{c}\text { Quant. de } \\
\text { Estudos }\end{array}$ \\
\hline $\begin{array}{c}\text { Gonçalves et al. } \\
(2019)\end{array}$ & Manual & 7 & TEA & Sim & 17 \\
\hline $\begin{array}{c}\text { Freitas e Souza } \\
(2018)\end{array}$ & Automática & 3 & Visual & Não & 40 \\
\hline Neto (2015) & Manual & 2 & $\begin{array}{c}\text { Sem } \\
\text { restrições }\end{array}$ & Não & 40 \\
\hline Este Trabalho & $\begin{array}{c}\text { Manual e } \\
\text { Automática }\end{array}$ & $\mathbf{8}$ & $\begin{array}{c}\text { Sem } \\
\text { restrições }\end{array}$ & Sim & $\mathbf{1 0 4}$ \\
\hline
\end{tabular}

\section{Mapeamento Sistemático da Literatura}

Neste trabalho, optou-se pela condução de um MSL, o mesmo diferente da revisão sistemática na qual possui um objetivo e questões de pesquisa muito específicas, o MSL apresenta como característica uma visão mais abrangente e questões de caráter exploratório sobre a área estudada [Petersen et al. 2015]. A Figura 1 ilustra as etapas e atividades realizadas. Estas são detalhadas nas próximas subseções que se seguem.

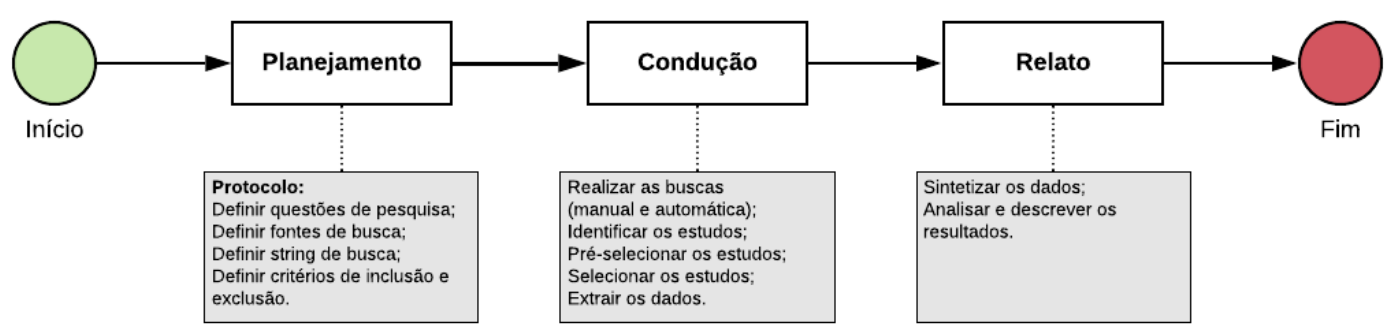

Figura 1. Processo de condução do MSL (adaptado [Petersen et al. 2015]).

\subsection{Questões de Pesquisa}

Inicialmente, elaborou-se o protocolo do MSL, o qual, junto com as demais atividades deste trabalho, foram realizadas pelo primeiro autor e, na sequência, revisadas pelos demais autores. Com isso, o MSL foi elaborado com o objetivo em responder a questão de pesquisa principal abordada nesta investigação, assim definida: "Como a tecnologia assistiva auxilia na inclusão educacional da pessoa com deficiência?". Para responder a questão de pesquisa, derivamos quatro subquestões específicas (QE), sendo elas:

QE1 - A qual público as tecnologias assistivas se destinam?

QE2 - Quais os tipos de soluções propostas pelos autores nos estudos primários?

QE3 - Quais estudos realizam comparações entre tecnologias assistivas? 
IX Congresso Brasileiro de Informática na Educação (CBIE 2020)

Anais do XXXI Simpósio Brasileiro de Informática na Educação (SBIE 2020)

QE4 - Qual a distribuição dos estudos em relação ao nível de ensino?

QE5 - Quais benefícios e dificuldades foram observados com o uso da TA na escola?

\subsection{Fontes e String de Busca}

Na sequência, foram definidas as fontes e string de busca. Considerando a busca manual, optou-se por eventos e revistas de destaque na área de informática na educação, como segue: Simpósio Brasileiro de Informática na Educação (SBIE), Workshop de Informática na Escola (WIE), Revista Brasileira de Informática na Educação (RBIE) e, a Revista Novas Tecnologias na Educação (RENOTE). No processo da busca automática, as bibliotecas digitais escolhidas foram utilizadas por possuírem relevância na academia, bem como disponibilidade para downloads de estudos primários, em especial, estudos escritos em língua portuguesa, sendo elas: Portal de Periódicos CAPES, Google Scholar, Scientific Electronic Library Online (SciELO) e, o Portal de Busca Integrada (SIBiUSP).

Para definição da string (Tabela 2) utilizada na busca automática, teve como base as questões de pesquisa. As palavras-chave "Tecnologia Assistiva" e "Educacional" foram utilizadas como termos principais, além dos seus respectivos termos alternativos.

Tabela 2. String de busca.

\begin{tabular}{|c|}
\hline String \\
\hline 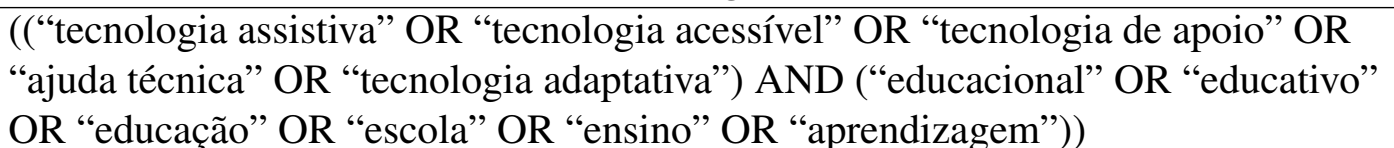 \\
\hline
\end{tabular}

\subsection{Seleção dos Estudos}

Por fim, Critérios de Inclusão (CI) e de Exclusão (CE) foram definidos visando analisar a relevância dos estudos retornados das buscas. Os CI foram: (i) estudos primários publicados entre 2010 e 2019; (ii) estudos primários escritos em língua portuguesa e (iii) estudos primários que apresentem o uso da TA na inclusão educacional da PcD. Como CE, definiu-se: (i) o estudo primário não está disponível online; (ii) estudos secundários (revisões e mapeamentos) e (iii) o estudo é uma versão antiga de outro já considerado.

Na segunda etapa, a busca inicial resultou em 12.279 estudos primários. Logo após, foram aplicados os CI e CE, resultando um total de 480 trabalhos que foram préselecionados e tiveram seus títulos, resumos e palavras-chave analisados. Na etapa de seleção, a leitura completa dos estudos foi realizada almejando identificar quais destes respondiam às questões de pesquisa. Ao fim, 104 estudos foram retornados ao conjunto resultante deste MSL, os mesmos seguem disponíveis online ${ }^{1}$.

\subsection{Extração dos Dados}

$\mathrm{Na}$ extração dos dados, buscou-se obter informações para responder às questões de pesquisa, sendo utilizada uma planilha eletrônica para organizar os dados. As informações extraídas dos 104 estudos foram: (i) deficiência abordada; (ii) tipo de solução proposta pelo próprio autor do estudo; (iii) comparação realizada entre recursos de TA (caso tenha

\footnotetext{
${ }^{1}$ https://bit.ly/EstudosSelecionados
} 
estabelecido); (iv) níveis de ensino e disciplinas abordadas e (v) benefícios e dificuldades ao utilizar a TA em sala de aula. A partir dos dados extraídos, iniciou-se o processo de interpretação dos resultados, elaboração de tabelas e gráficos para apresentação e relato das informações. Vale ressaltar que, neste trabalho não foi realizada a avaliação de qualidade, levando em consideração que não apresentaria altos requisitos nos estudos, pois o objetivo do MSL é fornecer uma visão geral da área estudada [Kitchenham et al. 2010].

\section{Relato e Discussão dos Resultados}

Nessa Seção, são apresentados e discutidos os resultados para as questões especificas de pesquisa apresentadas anteriormente.

\subsection{QE1 - A qual público as tecnologias assistivas se destinam?}

Todas as categorias de deficiência seguindo a classificação do Instituto Brasileiro de Geografia e Estatística (IBGE) foram citadas entre os estudos, porém, mais da metade das publicações são destinadas a PcD visual e auditiva, como mostra a Figura 2.

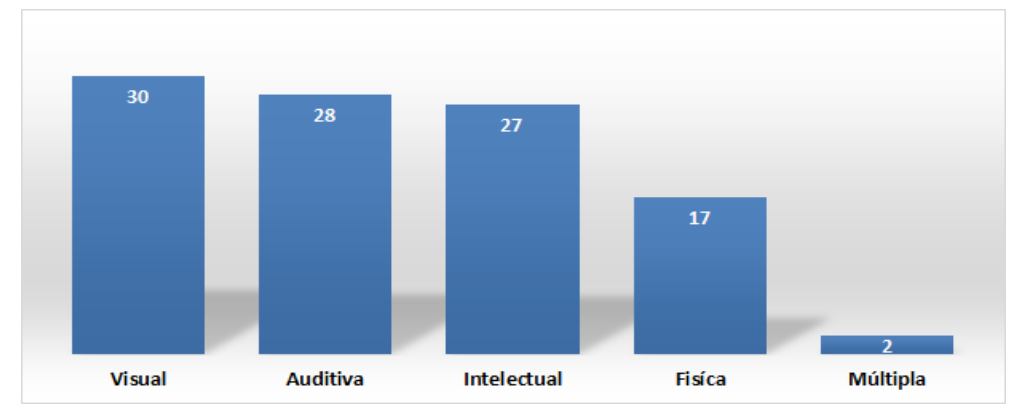

Figura 2. Deficiências apontadas pelos estudos primários avaliados.

Os estudos primários referentes à deficiência intelectual apresentavam detalhadamente os seus subtipos, de modo que, dentre os 27 estudos, 11 são voltados a pessoas com necessidades educacionais específicas, 10 relacionados ao TEA, 4 estudos sobre a síndrome de down e 2 voltados a pessoas com dislexia. Vale ressaltar que, para alguns especialistas, o TEA não necessariamente está relacionado a esse tipo de deficiência, uma pessoa autista pode ou não apresentar comprometimento intelectual [Orrú 2006].

\subsection{QE2 - Quais os tipos de soluções propostas pelos autores nos estudos primários?}

A análise realizada nos estudos primários indicou uma grande variedade de soluções nesta perspectiva. Estas foram agrupadas de acordo com a classificação definida pelo próprio autor, como mostra a Figura 3. Percebe-se que as soluções mais propostas são softwares e ferramentas educativas, isto acontece mediante a possibilidade oferecida pelos computadores em auxiliar o ensino da PcD ao utilizar recursos acessíveis. Além disso, na mesma Figura é possível identificar a quantidade de soluções propostas por tipo de deficiência.

Essa questão ainda identificou os recursos digitais de TA mais citados entre o conjunto de estudos avaliados. A Tabela 3 apresenta a lista com relação a essa vertente. 

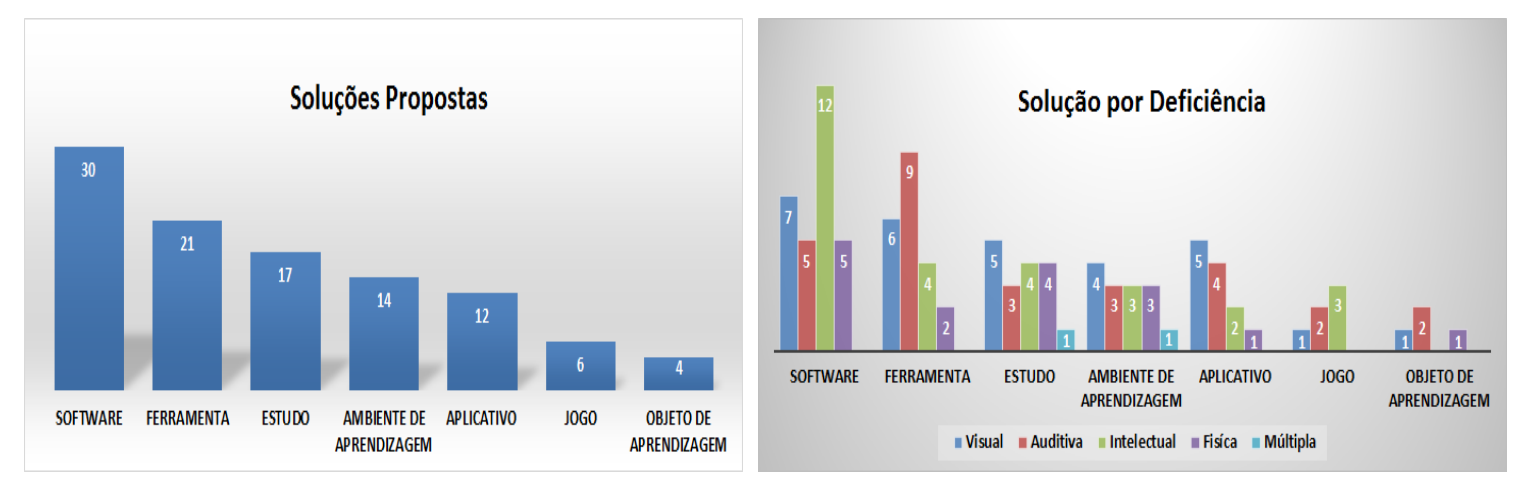

Figura 3. Soluções propostas com o uso da tecnologia assistiva.

Tabela 3. Principais recursos de tecnologia assistiva por deficiência.

\begin{tabular}{l|l}
\hline Deficiência & \multicolumn{1}{c}{ Recurso } \\
\hline Visual & DOSVOX - NVDA - Contátil \\
\hline Auditiva & ProDeaf - HandTalk - Libras Brincando e Aprendendo \\
\hline Física & Picture Communication Symbols - Vox4all - SCALA \\
\hline Intelectual & SCALA - MoviLetrando \\
\hline
\end{tabular}

\subsection{QE3 - Quais estudos realizam comparações entre tecnologias assistivas?}

Foram identificados três estudos primários que realizaram comparações entre recursos de tecnologia assistiva. Os mesmos tinham como objetivo apresentar para cada recurso os seus benefícios, limitações e o contexto em que melhor adéquam-se. Com isso, os estudos enquadram-se como um exemplo de serviço prestado à pessoa com deficiência, auxiliando na decisão de escolha sobre qual recurso utilizar. Os estudos selecionados foram:

EP11: realizou uma análise entre a tecnologia impressa do Braille, e o software Dosvox. Após observar os testes realizados utilizando um aluno com deficiência física e visual do $6^{\circ}$ ano, a professora responsável levantou critérios para análise: (i) o conforto é traduzido através do peso da máquina Perkins e o esforço para digitar nela, em contraste com a leveza do notebook para utilização do Dosvox; (ii) relatou que a introdução do Dosvox facilitava na realização das provas. Apesar de ser a favor da tecnologia digital, a mesma pondera que o Dosvox e o Braille têm prós, contras, e contextos que devem ser analisados.

EP48: analisou os aplicativos HandTalk e ProDeaf, ambos tradutores da língua portuguesa para Libras. Foram expostas características destes recursos, destacando: (i) ambos estão disponíveis para Android e IOS, mas apenas o HandTalk para Windows Phone; (ii) ambos realizam a tradução a partir da entrada de texto e voz; (iii) apenas o HandTalk permite girar o personagem para visualizar o sinal em diferentes ângulos. Ao final, percebeu-se que ambos são capazes de auxiliar na inclusão da pessoa surda.

EP59: realizou um estudo sobre aplicativos móveis a serem utilizados por deficientes visuais, sendo eles: (i) o Braille Play, um jogo que promove a alfabetização de crianças em braille; (ii) o BrailleTouch, que promove o aprendizado do alfabeto braille através da digitação em dispositivos móveis; (iii) o V-Braille, um aplicativo que ajuda as 
crianças e seus pais no exercício da escrita e leitura em braille.

\subsection{QE4 - Qual a distribuição dos estudos em relação ao nível de ensino?}

A Figura 4 apresenta como distribuiu-se o número de publicações por nível de ensino, sendo possível identificar que mais da metade dos estudos primários fazem referência ao ensino fundamental e ao Atendimento Educacional Especializado (AEE).

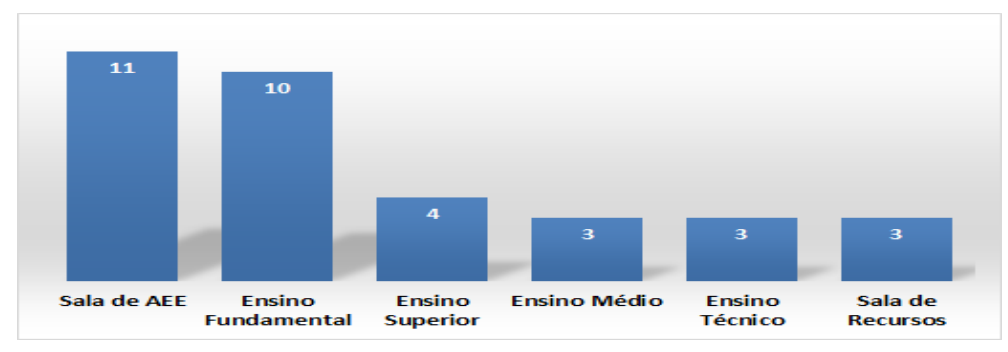

Figura 4. Tecnologias assistivas por nível de ensino.

Nesta mesma análise, foi possível constatar quais as disciplinas abordadas entre os estudos primários, identificando para quais delas os recursos de TA foram desenvolvidos, como ilustra a Figura 5. Destaque para as disciplinas de informática e matemática.

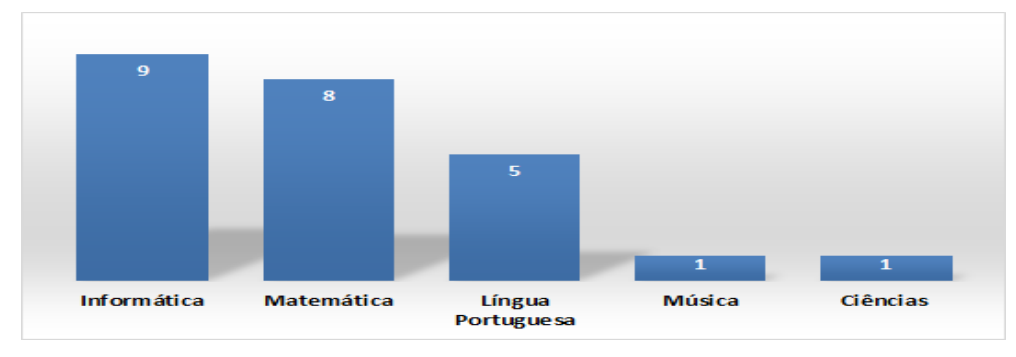

Figura 5. Tecnologias assistivas por disciplina.

\subsection{QE5 - Quais benefícios e dificuldades foram observados com o uso da TA na escola?}

Os relatos dos autores em 11 estudos, citam benefícios e dificuldades observados com o uso da TA em sala de aula, os quais foram feitos pelos educadores e educandos com deficiência. A Tabela 4 apresenta os benefícios observados com seus respectivos estudos.

Tabela 4. Benefícios identificados utilizar a tecnologia assistiva.

\begin{tabular}{c|l}
\hline Estudos & \multicolumn{1}{c}{ Benefícios } \\
\hline $\begin{array}{c}\text { EP21, EP26, EP30, } \\
\text { EP38 e EP75 }\end{array}$ & $\begin{array}{l}\text { Identificaram maior motivação em realizar as atividades por } \\
\text { parte dos alunos, além do aumento da autoestima. }\end{array}$ \\
\hline EP32 & $\begin{array}{l}\text { Os recursos desenvolvidos nos padrões de acessibilidade da } \\
\text { W3C, apresentaram menores dificuldades em seu uso. }\end{array}$ \\
\hline EP03 & $\begin{array}{l}\text { O uso em ambiente escolar, segundo os relatos dos educadores, } \\
\text { dá amostras de importantes avanços no aproveitamento escolar. }\end{array}$ \\
\hline
\end{tabular}


Em contrapartida, dificuldades foram observadas ao utilizar a TA, como mostra a Tabela 5. Concluindo que, ainda é uma área que vale a pena investir esforços em pesquisa.

Tabela 5. Dificuldades apontadas ao utilizar a tecnologia assistiva.

\begin{tabular}{c|l}
\hline Estudos & \multicolumn{1}{c}{ Dificuldades } \\
\hline EP11 & $\begin{array}{l}\text { Preocupação com a falta de responsabilidade dos jovens com os } \\
\text { estudos, deveres escolares e a apropriação da tecnologia proposta. }\end{array}$ \\
\hline EP28 e EP51 & $\begin{array}{l}\text { Falta a PcD, pais e professores, conhecimento nos recursos de TA, } \\
\text { considerando que muitos nunca tinham ouvido falar nessa tecnologia. }\end{array}$ \\
\hline EP46 & $\begin{array}{l}\text { Escassez de pesquisas relacionadas a inclusão digital de determinadas } \\
\text { deficiências, boa parte se encontra desprovida de TAs e recursos. }\end{array}$ \\
\hline
\end{tabular}

\subsection{Catálogo Online}

A partir da condução deste MSL, um catálogo online ${ }^{2}$ foi desenvolvido. O mesmo consiste em uma página WEB gratuita voltada para o âmbito educacional, com o objetivo em compartilhar recursos digitais de tecnologia assistiva para alunos com deficiência. Os recursos foram selecionados e catalogados dentre aqueles retornados da literatura. Estes, podem ser utilizados no processo de ensino e aprendizagem, fazendo com que a PcD realize suas atividades e aprenda de forma autônoma. Os recursos são organizados por deficiência, onde possuem informações pedagógicas e técnicas.

Ao observar a Figura 6, é possível identificar a primeira imagem a qual é referente a página inicial do catálogo, nela são ofertados a opção de acesso as definições sobre a TA (segunda imagem), além de conhecer os recursos digitais catalogados e disponíveis.

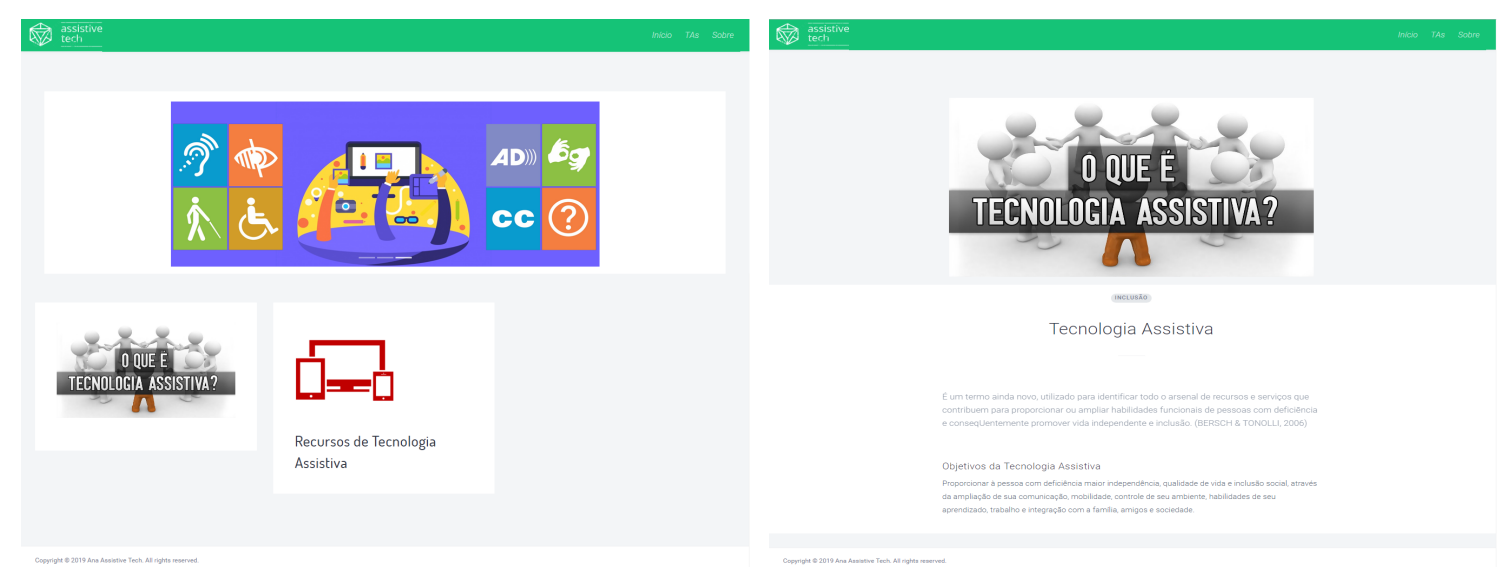

Figura 6. Páginas iniciais do catálogo desenvolvido.

Na primeira imagem da Figura 7 é observada a página que apresenta os recursos digitais de TA disponíveis. Estes podem ser filtrados de acordo com a deficiência, tipo de solução e a faixa etária do aluno. Ao selecionar um dos recursos, uma outra página é aberta contendo informações pedagógicas e técnicas como forma de auxiliar quem está buscando por uma tecnologia acessível, como ilustra a segunda imagem.

\footnotetext{
${ }^{2} \mathrm{https} / / / \mathrm{carolmelo.github.io/}$
} 


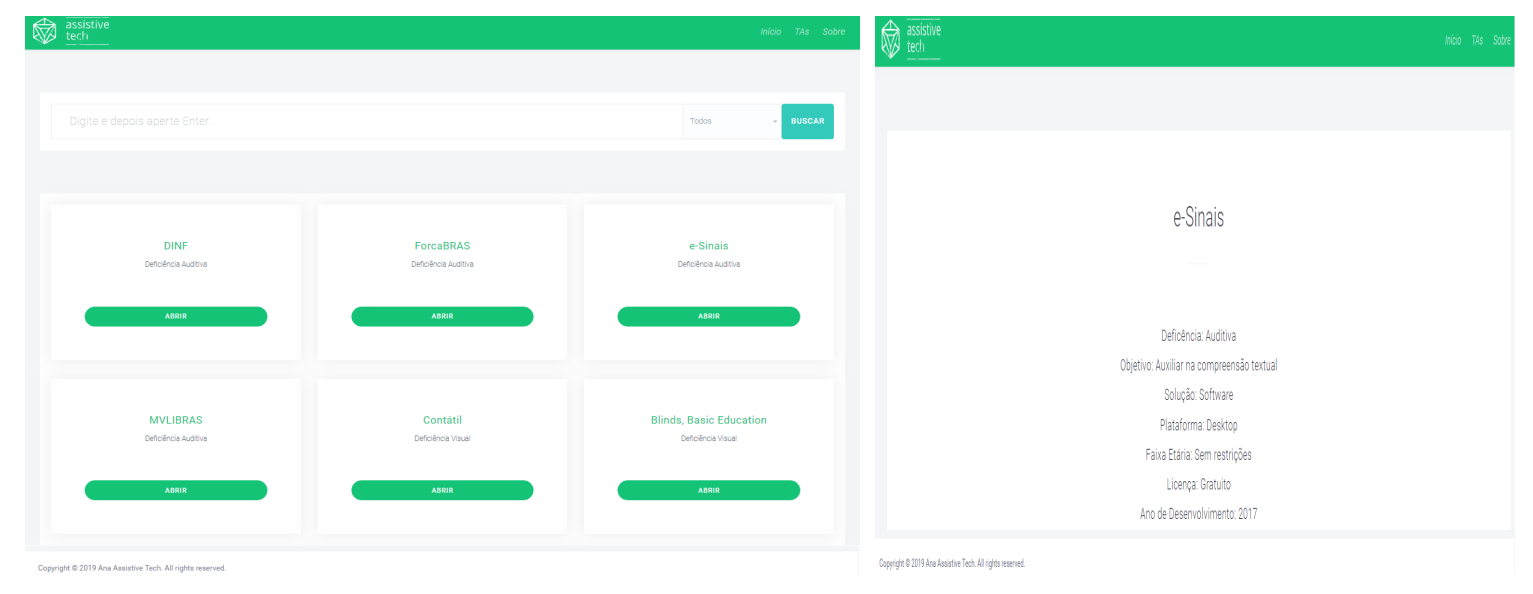

Figura 7. Páginas de acesso aos recursos digitais.

\section{Conclusão, Discussão e Trabalhos Futuros}

Este trabalho apresentou um MSL dos últimos 10 anos (2010 a 2019) do estado da arte em relação ao uso da tecnologia assistiva como auxílio a inclusão educacional da PcD. Ao final, 104 estudos foram selecionados, e todas as questões de pesquisa foram respondidas. Considerando a análise dos estudos, todas as deficiências seguindo a classificação do IBGE foram citadas, mas cerca de $55 \%$ das publicações atendem as deficiências visual e auditiva. Na sequência, um total de sete soluções propostas foram identificadas, dentre estas soluções, destaque para softwares e ferramentas educativas. Na mesma questão, foram identificadas para quais deficiências estas soluções estão voltadas, com isso, é possível analisar a ausência de determinadas tecnologias a pessoas com necessidades específicas.

Na sequência, identificou-se que três estudos realizavam comparações entre recursos de TA, e possuíam como objetivo apresentar os benefícios, limitações e em qual situação cada recurso melhor se encaixa. Mas, em contrapartida, ainda existe uma ausência nesse tipo de análise, principalmente voltadas as deficiências especificas, o que torna-se uma oportunidade para novas investigações. Em seguida, uma análise em relação a como se distribuiu o número de publicações por nível de ensino foi realizada e, nesta mesma questão, recursos específicos estão voltados a auxiliar na aprendizagem de determinadas disciplinas, a exemplo, informática e matemática. Contudo, muitos conteúdos não foram abordados, o que se torna uma lacuna no processo de ensino. Por fim, relatos de educadores e alunos retratando os benefícios e desafios ao utilizar a TA em sala de aula foram colhidos, mas nota-se uma ausência de relatos de experiência, considerando que poucos trabalhos realizaram estudos de caso e, aplicação dos recursos na prática.

Também foram selecionados e catalogados os recursos digitais de TA retornados da literatura, os mesmos foram organizados por tipo de deficiência, buscando destacar os pontos de interesse e sua contribuição diante do contexto abordado. Essas evidências auxiliaram no desenvolvimento do catálogo, que teve como objetivo auxiliar no processo de ensino e aprendizagem, fazendo com que a pessoa com deficiência realize suas atividades e aprenda de forma autônoma e estimulando suas habilidades. Além de se tornar um instrumento com informações úteis a quem está em busca de uma ferramenta acessíveis, mas que nem sempre possui conhecimento das tecnologias existentes. 
Ao final, este trabalho apresentou como principal contribuição uma visão geral, não enviesada e passível de replicação do estado da arte dos recursos e serviços de TA desenvolvidos para auxiliar na inclusão educacional da PcD. Neste sentido, seus resultados são úteis tanto para os educadores quanto as pessoas com deficiência que necessitam fazer uso de tais recursos, como também aos pesquisadores que desejam desenvolver outros tipos de tecnologias no âmbito educacional, em especial voltados as deficiências nas quais ainda há escassez de soluções propostas, como aquelas apresentadas neste trabalho.

Atividades foram pensadas como continuidade para trabalhos futuros, sendo elas: (i) aplicação da string de busca em outras bibliotecas digitais, junto a busca manual em outras conferências e revistas da área, como também a realização do método snowball; (ii) análise de estudos publicados em língua inglesa; (iii) novas discussões deverão ser criadas para ampliar o entendimento sobre o tema, em especial sobre àquelas deficiências menos exploradas na última década e (iv) por fim, ampliação e divulgação do catálogo desenvolvido, melhorando as funcionalidades, organização e inserção de novas informações.

\section{Referências}

Alves, G. M. and Aguiar, Y. P. C. (2014). Acessibilidade e tecnologia assistiva no ambiente educacional: Mapeamento sistemático. In Anais do Workshop de Informática na Escola, volume 20, page 16.

Bersch, R. (2017). Introdução à tecnologia assistiva. 2017.

Gonçalves, R., Pessoa, C., Passos, O. M., and de Amorim, R. (2019). Ferramentas assistivas no ensino e aprendizagem de crianças com aspectro autista: Um mapeamento sistemático. In Brazilian Symposium on Computers in Education (Simpósio Brasileiro de Informática na Educação-SBIE), volume 30, page 1291.

Kitchenham, B. A., Budgen, D., and Brereton, O. P. (2010). The value of mapping studies-a participant-observer case study. In 14th international conference on evaluation and assessment in software engineering (ease), pages 1-9.

Neto, L. and de Souza, H. (2015). Abordagens para inclusão educacional e em ambientes de trabalho de pessoas com deficiência: um mapeamento sistemático em eventos brasileiros.

Orrú, S. (2006). A constituição da linguagem de alunos autistas apoiada em comunicação suplementar alternativa. Universidade Metodista de Piracicaba, Faculdade de Ciências Humanas. Programa de Pós-graduação em Educação. Piracicaba.

Petersen, K., Vakkalanka, S., and Kuzniarz, L. (2015). Guidelines for conducting systematic mapping studies in software engineering: An update. Information and Software Technology, 64:1-18.

Souza, A. and Freitas, D. (2018). Tecnologias assistivas para apoiar o ensino e aprendizagem de pessoas com deficiência visual na matemática: Uma revisão sistemática da literatura. In Brazilian Symposium on Computers in Education (Simpósio Brasileiro de Informática na Educação-SBIE), volume 29, page 923.

Wohlin, C., Runeson, P., Neto, P. A. d. M. S., Engström, E., do Carmo Machado, I., and De Almeida, E. S. (2013). On the reliability of mapping studies in software engineering. Journal of Systems and Software, 86(10):2594-2610. 